\title{
mp-MRI Prostate Characterised PIRADS 3 Lesions are Associated with a Low Risk of Clinically Significant Prostate Cancer - A Retrospective Review of 92 Biopsied PIRADS 3 Lesions
}

\author{
Heath Liddell ${ }^{a} \quad$ Rajeev Jyoti ${ }^{b} \quad$ Hodo Z. Haxhimolla ${ }^{a, c}$ \\ aDepartment of Urology, The Canberra Hospital, Garran; 'buniversal Medical Imaging, Calvary Hospital, Bruce; \\ 'The Australian National University, Garran, Australia
}

\section{Key Words \\ Image guided biopsy • Prostate neoplasms • \\ Prostate risk assessment $\cdot$ Magnetic resonance imaging}

\begin{abstract}
Objective: To determine whether prostate image reporting and data system (PIRADS) 3 lesions as assessed by a 3T multiparametric magnetic resonance imaging (MRI) represent clinically significant prostate cancer. Method: A retrospective review was performed on a series of consecutive patients who underwent MRI guided biopsy of the prostate for clinical suspicion of prostate cancer between January 2013 and March 2014. Demographic, clinical, MRI and biopsy data were reviewed and compared. The same 3T MRI without the use of an endo-rectal coil was employed to assess each patient, obtaining high resolution $\mathrm{T} 2$ weighted images, diffusion weighted imaging and dynamic contrast enhancement. The MRI data was sent to Dynacad software for analysis. A single experienced radiologist reported all the studies from this series using a modified PIRADS scoring system. Subsequently, all the lesions marked PIRADS 3 or above were targeted with 18G core biopsy using DynaTrim in-gantry MRI guidance system. Needle position targeting the lesion was recorded prior to each biopsy. All core biopsy samples were sent to one of two pathology laboratories where they were processed and reported as per the International Society of
\end{abstract}

\section{KARGER}

Fax +4161306 1234

E-Mail karger@karger.ch

www.karger.com
(C) 2014 S. Karger AG, Basel

1015-9770/14/0082-0096\$38.00/0

Accessible online at:

www.karger.com/cur
Urological Pathology protocols. Results: One hundred and eighteen patients comprising a total of 215 lesions were reviewed. Amongst this cohort, 92 PIRADS 3 lesions were identified and biopsied. The mean age of patients in this cohort was 62.6 years. Median prostate specific antigen (PSA) was $6.5 \mathrm{ng} / \mathrm{ml}$ and median prostate size was $78.4 \mathrm{ml}$. Eightysix (93.5\%) of biopsied PIRADS 3 lesions were benign and 6 (6.5\%) lesions were found to be malignant. Of these 6 malignant lesions, $4(66 \%)$ were Gleason score $6(3+3)$ and 2 $(33 \%)$ were Gleason score $7(3+4)$. Of the 86 non-malignant lesions, $1(1.2 \%)$ represented high-grade prostate intraepithelial neoplasia and 2 (2.4\%) represented atypical small acinar proliferation. PIRADS 3 lesions within the peripheral zone were more likely to be associated with malignant disease compared with lesions identified within the transition zone (10.8 vs. $3.8 \%)$. Those with malignant disease had a higher median PSA (8.1 vs. $6.4 \mathrm{ng} / \mathrm{ml}$ ) and higher median PSA density ( 0.12 vs. 0.08 ) than those without malignant disease. Those with benign pathology had a higher prevalence of inflammation (31.4 vs. 16.7\%). As per Epstein's criteria, $4(4.3 \%)$ of the biopsied lesions represented clinically significant disease. Conclusion: We have demonstrated in our series, that prostate lesions characterized on a $3 \mathrm{~T}$ multiparametric MRI examination of the prostate as PIRADS 3, according to the current prevalent scoring systems, are associated with a low likelihood of the presence of clinically significant prostate cancer.

Copyright $\odot 2014$ S. Karger AG, Basel 


\section{Introduction}

Multiparametric magnetic resonance imaging (mpMRI) is now gaining widespread use amongst urologists for the diagnosis, surveillance and staging of patients with prostate cancer [1]. With a high negative predictive value of $80-90 \%$, many patients with high-risk features [abnormal prostate specific antigen (PSA), suspicious digital rectal examination (DRE), strong family history of prostate cancer] are being offered an MRI to assess for the presence of prostate cancer prior to transrectal and transperineal ultrasound guided biopsies in the first instance [2]. European Society of Urogenital Radiology (ESUR) has developed guidelines that describe a structured reporting system, prostate imaging reporting and data system (PIRADS), for the assessment of lesions suspicious for prostate cancer on MRI [3]. This system is based upon a 'Likert scale' whereby lesions are scored from 1 to 5 based upon the various MRI parameter characteristics they display. Lesions scored 1 or 2 are considered to indicate that clinically significant cancer is unlikely to be present. Lesions scored 4 or 5 are considered to indicate that clinically significant cancer is likely to be present. PIRADS 3 lesions are equivocal for the presence of prostate cancer and pose a significant clinical management challenge. We set out to determine whether PIRADS 3 lesions as assessed by a 3T mpMRI represent clinically significant prostate cancer.

\section{Subjects and Methods}

After institutional approval was obtained, a retrospective review was performed on a series of consecutive patients who underwent MRI guided biopsy of the prostate for clinical suspicion of prostate cancer (either elevated PSA, abnormal DRE or both) between January 2013 and March 2014. Demographic, clinical, MRI and biopsy data were reviewed. Demographic data included the patients' age. Clinical data included the PSA value, PSA density and free to total ratio if performed. MRI data included prostate volume; location of lesion; T2 weighted images (T2WI), diffusion weighted imaging (DWI) and dynamic contrast enhancement (DCE) score and overall PIRADS score. Biopsy data included presence of malignancy, Gleason score, number of cores positive, percentage of cores positive, presence of perineural invasion, lymphovascular invasion, extraprostatic spread, inflammation, high-grade prostatic intraepithelial neoplasia (HGPIN) and atypical small acinar proliferation (ASAP).

The same 3T MRI (Philips Ingenia 3.0T) without the use of an endo-rectal coil was employed to assess each patient, obtaining high resolution T2WI, DWI and DCE. T1 images were also obtained to detect any hemorrhage, calcification or pelvic lymph nodes. The MRI data was sent to Dynacad (Invivo Inc) software for analysis. A single radiologist with over 6 years experience
Table 1. Demographics

\begin{tabular}{ll} 
Characteristic & Value \\
\hline Number of patients & 118 \\
Number of lesions & 215 \\
Age (mean \pm SD) (year) & $62.2 \pm 6.7$ \\
Median PSA (ng/ml) & 6.5 \\
Median prostate volume (ml) & 78.4 \\
\hline
\end{tabular}

Table 2. Characteristics of malignant PIRADS 3 lesions

\begin{tabular}{ll}
\hline Characteristic & Value \\
\hline Number of patients & 4 \\
Number of lesions & 6 \\
Median PSA $(\mathrm{ng} / \mathrm{ml})$ & 8.1 \\
Prostate volume $(\mathrm{ml})$ & 60.1 \\
Median PSA density $(\mathrm{ng} / \mathrm{ml} / \mathrm{ml})$ & 0.12 \\
Location of lesion & \\
$\quad$ Central zone & 0 \\
$\quad$ Peripheral zone & 4 \\
Transition zone & 2 \\
Gleason 3 + 3 = 6 & 4 \\
Gleason 3 + 4 7 & 2 \\
Number of lesions qualify as insignificant cancer & 2 \\
ASAP & 0 \\
HGPIN & 0 \\
Inflammation & 1
\end{tabular}

in prostate MRI reported all the studies from this series using a modified PIRADS scoring system. Subsequently, all the lesions marked PIRADS 3 or above were targeted with $18 \mathrm{G}$ core biopsy using DynaTrim (Invivo Inc) in-gantry MRI guidance system. Needle position targeting the lesion was recorded prior to each biopsy, as per system specifications. All core biopsy samples were sent to one of two pathology laboratories where they were processed and reported as per the International Society of Urological Pathology protocols.

\section{Results}

One hundred and eighteen patients comprising a total of 215 lesions were reviewed. Amongst this cohort, 92 PIRADS 3 lesions were identified and biopsied. The mean age of patients was 62.6 years. Median PSA was $6.5 \mathrm{ng} / \mathrm{ml}$ and median prostate size was $78.4 \mathrm{ml}$ (table 1). Eighty-six (93.5\%) of the biopsied PIRADS 3 lesions were benign and $6(6.5 \%)$ of these lesions were found 
Table 3. Characteristics of non-malignant PIRADS 3 lesions

\begin{tabular}{ll} 
Characteristic & Value \\
\hline Number of patients & 61 \\
Number of lesions & 86 \\
Median PSA $(\mathrm{ng} / \mathrm{ml})$ & 6.4 \\
Prostate volume $(\mathrm{ml})$ & 84.5 \\
Median PSA density $(\mathrm{ng} / \mathrm{ml} / \mathrm{ml})$ & 0.08 \\
Location of lesion & \\
$\quad$ Central zone & 2 \\
$\quad$ Peripheral zone & 33 \\
$\quad$ Transition zone & 51 \\
ASAP & 2 \\
HGPIN & 1 \\
Inflammation & 27 \\
\hline
\end{tabular}

Table 4. PIRADS score

\begin{tabular}{ll}
\hline Score & Significance \\
\hline 1 & $\begin{array}{l}\text { Clinically significant disease is highly unlikely } \\
\text { to be present } \\
\text { Clinically significant disease is unlikely to be } \\
\text { present }\end{array}$ \\
3 & $\begin{array}{l}\text { Clinically significant cancer is equivocal } \\
\text { Clinically significant cancer is likely to be } \\
\text { present } \\
\text { Clinically significant cancer is highly likely to } \\
\text { be present }\end{array}$ \\
\hline
\end{tabular}

on histological analysis to be prostatic adenocarcinoma. Of these 6 prostatic adenocarcinoma's that were identified, $4(66 \%)$ lesions were Gleason score $6(3+3)$ and 2 $(33 \%)$ lesions were Gleason score $7(3+4)$. Of the 6 malignant PIRADS 3 lesions, 4 (66\%) were located within the peripheral zone of the prostate and $2(33 \%)$ were located within the transition zone (table 2). Of the 86 lesions that were not malignant $1(1.2 \%)$ had evidence of HGPIN, 2 (2.4\%) had evidence of ASAP and 27 (31.4\%) had evidence of inflammation as defined by macrophage or neutrophil infiltration. PIRADS 3 lesions that were not malignant were more likely to be found within the transition zone compared with the peripheral zone (51 vs. 33) and central zone (51 vs. 2) (table 3 ). Overall, $10.8 \%$ of PIRADS 3 lesions within the peripheral zone were associated with prostate adenocarcinoma compared with $3.8 \%$ of lesions located within the transition zone.
Those with malignant disease were noted to have a higher PSA and higher PSA density than those without malignant disease. Those with benign pathology also had a significantly higher prevalence of inflammation reported after histological analysis.

\section{Discussion}

The ESUR developed the PIRADS structured reporting system to allow for reproducible and reliable communication of mpMRI prostate data. The system employs the use of a Likert-like scoring system to indicate the likelihood of the presence of clinically significant disease (table 4). Once a region of interest is identified, a score from 1 to 5 is assigned to the lesion on the T2WI anatomical view and on at least two functional MRI techniques (DCE, DWI, and spectroscopy) as outlined in table 5. An overall score is then given to the region of interest.

Although PIRADS is increasingly being adopted as a way of communicating mpMRI prostate results, its limitations are well recognized [4]. Most radiologists employ a modified PIRADS scoring technique when reporting mpMRI of the prostate whereby each lesion is not formally scored as per the PIRADS system. Instead, an analogous Likert-like score is assigned to a lesion based upon the combined characteristics of the lesion, but not strictly following the ESUR proposed guidelines due to its limitations. As can be seen from table 5, the characteristics of PIRADS 3 lesions are not well described. Even when the PIRADS system is strictly followed, the descriptor 'intermediate appearances, not in categories $1 / 2$ or $4 / 5$ ' has the potential to create significant inter-observer variability in the characterization of lesions and therefore potential variability in the correct identification of lesions that are clinically significant.

Such variability is particularly troublesome in determining the significance of PIRADS 3 lesions. It is generally well accepted that lesions scored as PIRADS 4 and 5 are likely to be associated with significant cancer and that intervention should be undertaken to obtain a histological diagnosis. Conversely so, PIRADS 1 and 2 lesions are likely to be associated with benign or insignificant disease and further intervention is not required to assess these lesions. The clinical significance of, and how to manage patients with PIRADS 3 lesions remains uncertain.

Amongst the literature, significant variability exists in the detection of prostatic adenocarcinoma amongst biopsied PIRADS 3 lesions. Pokorny et al. [5] prospectively 
Table 5. PIRADS scoring system

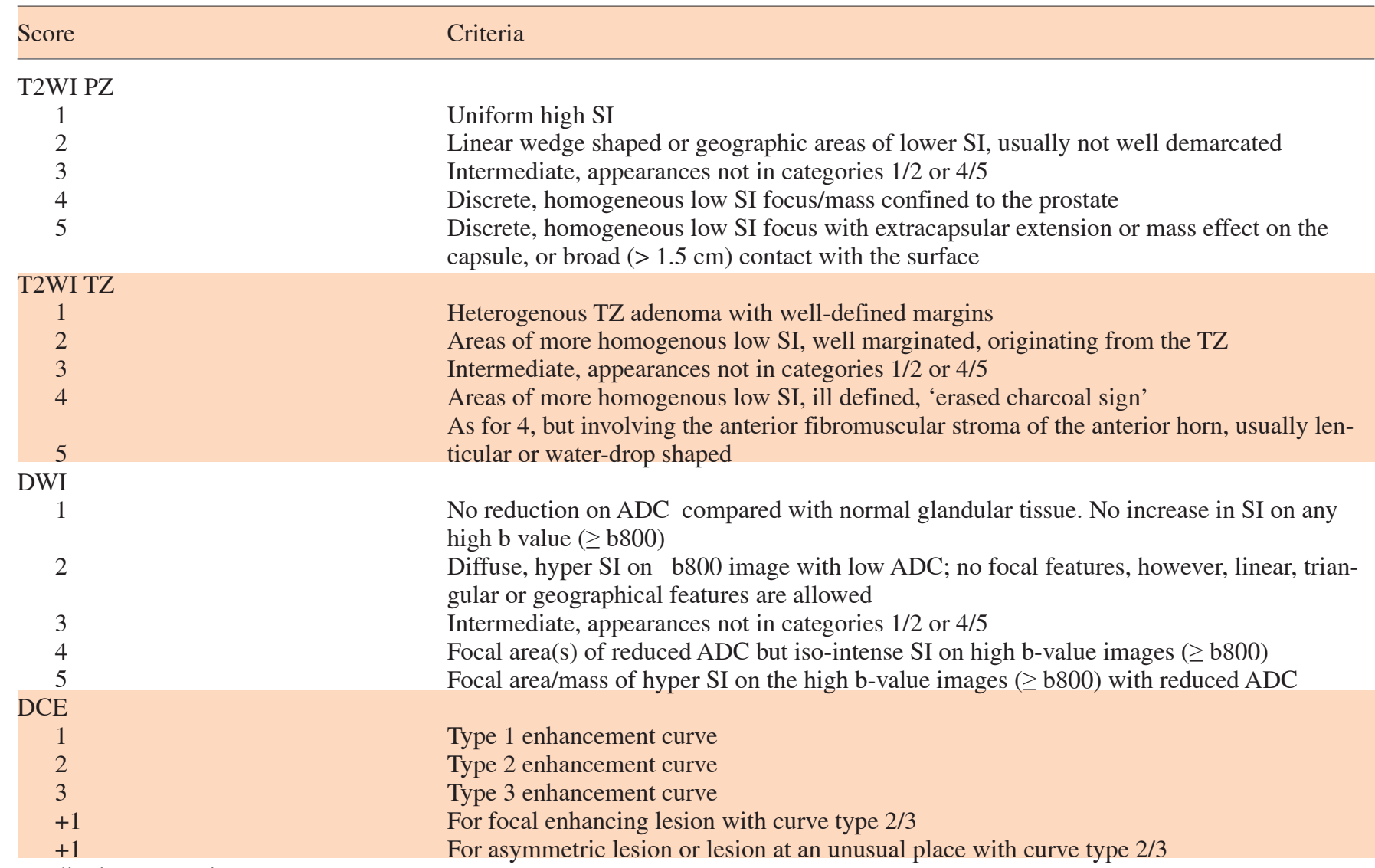

Qualitative magnetic resonance

stectroscopic imaging
1
2
3
4
5
Citrate peak level exceeds choline peak $>2$ times
Citrate peak level exceeds choline peak $>1,<2$ times
Citrate peak level equals choline peak height
Choline peak level exceeds citrate peak $>1,<2$ times
Choline peak level exceeds citrate peak $>1,<2$ times

$\mathrm{PZ}=$ Peripheral zone; $\mathrm{SI}=$ signal intensity; $\mathrm{TZ}=$ transition zone; $\mathrm{ADC}=$ apparent diffusion coefficient.

reviewed 226 patients who underwent a $3 \mathrm{~T}$ mpMRI for clinical suspicion of prostate cancer. Those with PIRADS 3-5 lesions underwent an MRI guided biopsy. In their series, 33 patients had PIRADS 3 lesions that were biopsied and of those, 5 (15\%) patients had histologically confirmed prostatic adenocarcinoma. Thompson et al. [6] reported a $26 \%$ detection rate of prostatic adenocarcinoma amongst their series of biopsied PIRADS 3 lesions. Amongst these, $38 \%$ were moderate- or high-risk lesions.

In the current study, we report our experience of 92 biopsied PIRADS 3 lesions. We found that our detection rate of prostatic adenocarcinoma amongst these lesions to be $6.5 \%$. If one was to apply Epstein's criteria for insignificant disease to MRI guided biopsies (no Gleason pattern 4 or 5 , less than 3 core samples involved of a sextant biopsy, and no core sample $>50 \%$ involved), $2.1 \%$ of patients in our series were found to have an insignificant cancer whereas $4.4 \%$ were found to have significant disease $[7,8]$. Of those with benign disease $(93.5 \%), 31 \%$ were found to have significant histopathological evidence of inflammation. They also had a lower median PSA and a higher mean prostate volume. We also noted that PIRADS 3 lesions identified within the transition zone were more likely to represent benign disease compared with PIRADS 3 lesions identified within the peripheral zone. 
To our knowledge, this is the largest study assessing MRI guided biopsy of PIRADS 3 lesions. In our series, we have attempted to confirm the significance of PIRADS 3 lesions so that appropriate clinical decisions can be made regarding the future management of patients presenting with such lesions. Given the wide variation in the literature of the prevalence of prostate carcinoma associated with biopsied PIRADS 3 lesions, we believe that current reporting does not allow for a generalized guideline on how to manage such patients. In our series, we have demonstrated that PIRADS 3 lesions as reported in our institution are associated with a low risk of prostate cancer and thus are suitable for ongoing surveillance. Our paper contributes to the current body of evidence regarding the significance of biopsied PIRADS 3 lesions and highlights that inter-observer variability exists in the characterization of these lesions and thus the subsequent likelihood of the presence of prostate cancer. Our study also highlights the importance of auditing ones own local data so that the clinical significance of reported prostate MRI data is known to help inform appropriate clinical decision-making. As reporting systems are further developed and refined, the variability in the reporting of what constitutes a clinically significant lesion should decrease and therefore appropriate guidelines will then be able to be developed to help guide management of patients undergoing mpMRI prostate.

\section{Conclusion}

We have demonstrated in our series, that prostate lesions characterized on a $3 \mathrm{~T}$ mpMRI examination of the prostate as PIRADS 3, according to the current prevalent scoring systems, are associated with a low likelihood of the presence of clinically significant prostate cancer.

\section{References}

$>1$ Lee DJ, Ahmed HU, Moore CM, Emberton M, Ehdaie B: Multiparametric magnetic resonance imaging in the management and diagnosis of prostate cancer: current applications and strategies. Curr Urol Rep 2014;15:390.

-2 Kuru T, Roethke M, Rieker P, Roth W, Fenchel M, Hohenfellner M, Schlemmer H, Hadaschik B: Histology core-specific evaluation of the European Society of Urogenital Radiology (ESUR) standardised scoring system of multiparametric magnetic resonance imaging (mpMRI) of the prostate. BJU Int 2013;112:1080-1087.

-3 Barentsz JO, Richenberg J, Clements R, Choyke P, Verma S, Villeirs G, Rouviere O, Logager V, Futterer JJ: ESUR prostate MR guidelines 2012. Eur Radiol 2012;22:746757.
-4 Junker D, Schafer G, Edlinger M, Kremser C, Bektic J, Horninger W, Jaschke W, Aigner F: Evaluation of the PI-RADS scoring system for classifying mpMRI findings in men with suspicion of prostate cancer. Biomed Res Int 2013;2013:252939.

5 Pokorny MR, de Rooij M, Duncan E, Schröder FH, Parkinson R, Barentsz JO, Thompson LC: Prospective study of diagnostic accuracy comparing prostate cancer detection by transrectal ultrasound-guided biopsy versus magnetic resonance (MR) imaging with subsequent MR-guided biopsy in men without previous prostate biopsies. Eur Urol 2014;66:22-29.
6 Thompson J, Lawrentschuk N, Frydenberg M, Thompson L, Stricker P: The role of magnetic resonance imaging in the diagnosis and management of prostate cancer. BJU Int 2013;112(suppl 2):6-20.

$>7$ Epstein JI, Walsh PC, Carmichael M, Brendler CB: Pathologic and clinical findings to predict tumor extent of nonpalpable (stage T1c) prostate cancer. JAMA 1994;271:368374.

-8 Ploussard G, Epstein JI, Montironi R, Carroll PR, Wirth M, Grimm MO, Bjartell AS, Montorsi F, Freedland SJ, Erbersdobler A, van der Kwast TH: The contemporary concept of significant versus insignificant prostate cancer. Eur Urol 2011;60:291-303. 